\title{
THE DIMENSION OF THE CONVEX KERNEL AND POINTS OF LOCAL NONCONVEXITY
}

\author{
NICK M. STAVRAKAS
}

\begin{abstract}
Let $S$ be a compact connected subset of $R^{d}$. A necessary and sufficient condition is given to ensure that the dimension of the convex kernel of $S$ is greater than or equal to $k$, $0 \leqq k \leqq d$. This condition involves a visibility constraint on the points of local nonconvexity of $S$. As consequences, we obtain new characterizations of the convex kernel of $S$ and the $n$ th-order convex kernel of $S$.
\end{abstract}

1. Introduction. Since the convex kernel of a compact subset $S$ of $R^{d}$ is the intersection of the maximal convex subsets of $S$ and since there is a lack of information on the dimension of the intersection of an infinite collection of convex sets, it seems very hard in general to give strictly combinatorial conditions to ensure that the dimension of the convex kernel of $S$ is greater than or equal to $k, 0 \leqq k \leqq d$. This paper is an attempt to give a meaningful necessary and sufficient condition which is not combinatorial in nature. Specifically, we give a necessary and sufficient condition involving the points of local nonconvexity of $S$, whose proof depends heavily on the compactness of the points of local nonconvexity of $S$. For other results concerning the dimension of the convex kernel the reader should consult Kenelly and Hare [2], F. A. Toranzos [4], and Foland and Marr [1].

2. Notations and definitions. Let $S \subset R^{d}$. Let $A$ and $B$ be subsets of $S$. We say $A$ sees $B$ provided, for all $a \in A$ and $b \in B,[a b] \subset S$, where [ab] is the closed line segment from $a$ to $b$.

Definition 1. Let $S \subset R^{d}$. We say $x \in S$ is a point of local convexity of $S$ if there exists an open set $N_{x}$ containing $x$ such that $N_{x} \cap S$ is convex. If no such $N_{x}$ exists, $x$ is called a point of local nonconvexity of $S$.

Definition 2. Let $S \subset R^{d}$. The convex kernel of $S$ is the set $\{x \mid x \in S$ and $[x y] \subset S \forall y \in S\}$.

Definition 3. Let $S \subset R^{d}$. The nth order convex kernel of $S$ is the set of all $x \in S$ such that, for all $y \in S$, there exists a polygonal arc from $x$ to $y$ in $S$ consisting of $n$ or fewer line segments.

Received by the editors September 13, 1971.

AMS 1970 subject classifications. Primary 52A30; Secondary 52A20.

(C) American Mathematical Society 1972 
Definition 4. Let $H \subset R^{d}$. We say $H$ is a flat provided $H$ is the translate of a subspace of $R^{d}$.

The symbols $C(S)$ and $L(S)$ denote the points of local convexity of $S$ and the points of local nonconvexity of $S$, respectively. The symbols Ker $S$ and $K_{n}(S)$ denote the convex kernel of $S$ and the $n$th order convex kernel of $S$, respectively. The relative interior of $S$ with respect to a flat $H$ is denoted by rel int $(H \cap S)$.

3. The main result. Before proving our main theorem, we state the following result of Valentine [5] for later reference.

THEOREM 1. Let $S \subset R^{d}$ be compact and connected with $L(S) \neq \varnothing$. Then given $x \in S$ there exists $y \in L(S)$ such that $[x y] \subset S$.

The following theorem is the main result of this paper.

THEOREM 2. Let $S$ be a compact connected subset of $R^{d}$. Then

$$
\operatorname{dim}(\operatorname{Ker} S) \geqq k, \quad 0 \leqq k \leqq d,
$$

iff there exists a flat $H, \operatorname{dim} H=k$ and a point $x \in \operatorname{rel} \operatorname{int}(H \cap S)$ such that given $y \in L(S)$ there exists open sets $N_{y}$ and $N_{x}^{v}$ such that $N_{x}^{y} \cap S \cap H$ sees $N_{y} \cap S$.

Proof. The necessity part of the theorem is trivial. To prove sufficiency, we first note that $L(S)$ is compact since $S$ is. For each $y \in L(S)$ let $N_{x}^{y}$ and $N_{y}$ be as in the statement of the theorem. Then $\left\{N_{y} \mid y \in L(S)\right\}$ forms an open cover for $L(S)$. Thus select a finite subcover $\left\{N_{v_{1}}, \cdots, N_{y_{m}}\right\}$. Now since $x \in \operatorname{rel} \operatorname{int}(H \cap S)$, the set $\left(\bigcap_{i=1}^{m} N_{x}^{y_{i}}\right) \cap H \cap S$ contains a convex set $A$ with $\operatorname{dim} A=k$. Let $z$ be any element of $A$. We will show $z \in \operatorname{Ker} S$ and we will be done. Let $q \in S$. By Theorem 1 there exists $y \in L(S)$ such that $[y q] \subset S$. Since $y \in L(S), y \in N_{y_{i}}$ for some $i$. By hypothesis $z \in N_{x}^{y_{i}} \cap$ $H \cap S$ and $N_{x}^{y_{i}} \cap H \cap S$ sees $N_{y} \cap S$, so $z$ sees $N_{y_{i}} \cap S$, and so [zy] $\subset S$. Thus $l^{\prime}=[z y] \cup[y q]$ is an arc from $z$ to $q$ of finite arc length. Thus we may find an $\operatorname{arc} l^{\prime}$ from $z$ to $q$ in $S$ of minimal arc length. Let $l^{\prime}$ be the image of [01] by a continuous function $f$ with $f(0)=z$ and $f(1)=q$. Now we let $\alpha=$ $\sup \{\theta \mid \theta \in[01]$ and $f(\theta) \in L(S)\}$. We consider three cases:

Case 1. $\alpha=0$. Let $\left\{\alpha_{i}\right\}_{1}^{\infty}$ be a sequence with $0<\alpha_{i}<1$ and $\lim _{i \rightarrow \infty} \alpha_{i}=0$. Then $f\left(\left[\alpha_{i} 1\right]\right)$ is a minimal arc from $f\left(\alpha_{i}\right)$ to $f(1)=q$. Since $\alpha=0$ we have $f\left(\left[\alpha_{i} 1\right]\right) \subset C(S)$ and the minimality of $f\left(\left[\alpha_{i} 1\right]\right)$ implies that $f\left(\left[\alpha_{i} 1\right]\right)=$ $\left[f\left(\alpha_{i}\right) q\right]$. Now in the sense of the Hausdorff metric we have

$$
\lim _{i \rightarrow \infty} f\left(\left[\alpha_{i} 1\right]\right)=l^{\prime}
$$

and so $l^{\prime}=[z q]$.

Case 2. $0<\alpha<1$. By an argument similar to Case 1 , since $f([\alpha 1]) \subset$ $C(S)$ we have that $f([\alpha 1])=[f(\alpha) q]$. Since $L(S)$ is compact we have 
$f(\alpha) \in L(S)$. Thus $f(\alpha) \in N_{y_{j}}$ for some $j$. Now $z$ sees $N_{y_{j}} \cap S$, so $[z f(\alpha)] \subset S$ and the minimality of $l^{\prime}$ implies that $l^{\prime}=[z f(\alpha)] \cup[f(\alpha) q]$. Further, since $z$ sees $N_{y_{j}} \cap S$, the minimality of $l^{\prime}$ implies that $z, q$, and $f(\alpha)$ are all collinear, for if not we could shorten $l^{\prime}$, a contradiction. Thus $l^{\prime}=[z q]$.

Case 3. $\alpha=1$. Then $q \in L(S)$ and our hypothesis imply $[z q] \subset S$ and the theorem is established.

For $k=0$ we obtain the following characterization of $\operatorname{Ker} S$ from Theorem 2.

THEOREM 3. Let $S$ be a compact connected subset of $R^{d}$. Then $x \in \operatorname{Ker} S$ iff given $y \in L(S)$ there exists an open set $N_{y}$ such that $x$ sees $N_{y} \cap S$.

Sparks [3] has characterized $K_{n}(S)$, where $S \subset R^{2}$ is compact and simply connected as the intersection of the maximal $L_{n}$ subsets of $S$. Using a proof similar to the proof of Theorem 2, we may prove the following theorem:

THEOREM 4. Let $S$ be a compact connected subset of $R^{d}$. Then $x \in K_{n}(S)$ iff given $y \in L(S)$ there exists an open set $N_{y}$ such that, given $z \in N_{y} \cap S$, there exists a polygonal arc from $x$ to $z$ in $S$ consisting of $n$ or fewer line segments.

\section{BIBLIOGRAPHY}

1. N. E. Foland and J. M. Marr, Sets with zero-dimensional kernels, Pacific J. Math. 19 (1966), 429-432. MR 34 \#4983.

2. J. W. Kenelly, W. R. Hare et al., Convex components, extreme points and the convex kernel, Proc. Amer. Math. Soc. 21 (1969), 83-87.

3. A. Sparks, Characterizations of the generalized convex kernel, Proc. Amer. Math. Soc. 27 (1971), 563-566.

4. F. A. Toranzos, The dimension of the kernel of a starshaped set, Notices Amer. Math. Soc. 14 (1967), 832. Abstract \#67T-588.

5. F. A. Valentine, Local convexity and $L_{n}$ sets, Proc. Amer. Math. Soc. 16 (1965), 1305-1310. MR 32 \#2976.

Department of Mathematics, University of North Carolina at Charlotte, Charlotte, North Carolina 28213 